\title{
COMPARING THE RELATION BETWEEN STAR FORMATION AND GALAXY MASS IN DIFFERENT ENVIRONMENTS
}

\author{
Benedetta Vulcani ${ }^{1,2}$, Bianca M. Poggianti ${ }^{2}$, Rose A. Finn ${ }^{3}$, Gregory Rudnick ${ }^{4}$, Vandana Desai ${ }^{5}$, \\ AND STEVEN BAMFORD ${ }^{6}$ \\ ${ }^{1}$ Astronomical Department, Padova University, Padova, Italy \\ ${ }^{2}$ INAF-Astronomical Observatory of Padova, Padova, Italy \\ ${ }^{3}$ Department of Physics, Siena College, Loudonville, OH, USA \\ ${ }^{4}$ Department of Physics and Astronomy, University of Kansas, Lawrence, KS, USA \\ ${ }^{5}$ Spitzer Science Center, California Institute of Technology, Pasadena, CA, USA \\ ${ }^{6}$ School of Physics and Astronomy, University of Nottingham, Nottingham, UK \\ Received 2009 October 20; accepted 2009 December 4; published 2010 January 19
}

\begin{abstract}
Analyzing $24 \mu \mathrm{m}$ MIPS/Spitzer data and the [O II] 3727 line of a sample of galaxies at $0.4 \leqslant z \leqslant 0.8$ from the ESO Distant Cluster Survey, we investigate the ongoing star formation rate (SFR) and the specific star formation rate (SSFR) as a function of stellar mass in galaxy clusters and groups, and compare these with results from field studies. As for the field, we find a decline in SFR with time, indicating that star formation (SF) was more active in the past, and a decline in SSFR as galaxy stellar mass increases, showing that the current SF contributes more to the fractional growth of low-mass galaxies than high-mass galaxies. However, we find a lower median SFR (by a factor of $\sim 1.5$ ) in cluster star-forming galaxies than in the field. The difference is highly significant when all Spitzer and emission-line galaxies are considered, regardless of color. It remains significant at $z>0.6$ after removing red emission-line galaxies, to avoid possible active galactic nucleus contamination. While there is overlap between the cluster and field SFR-mass relations, we find a population of cluster galaxies $(10 \%-25 \%)$ with reduced SFR for their mass. These are likely to be in transition from star forming to passive. Separately comparing clusters and groups at $z>0.6$, only cluster trends are significantly different from the field, and the average cluster SFR at a given mass is $\sim$ two times lower than the field. We conclude that the average SFR in star-forming galaxies varies with galaxy environment at a fixed galaxy mass.
\end{abstract}

Key words: galaxies: clusters: general - galaxies: evolution - galaxies: formation

\section{INTRODUCTION}

The star formation (SF) activity, like other galaxy properties, varies systematically with galaxy mass and redshift. Its trend as a function of galaxy mass has been studied in the field at different epochs (e.g., Brinchmann et al. 2004; Noeske et al. 2007a; Elbaz et al. 2007; Daddi et al. 2007; Pannella et al. 2009). These works have found a strong and rather tight correlation between current star formation rate (SFR) and galaxy stellar mass for field star-forming galaxies at all redshifts out to $z=2$. This correlation shifts to progressively higher SFRs at higher $z$, remarkably maintaining its local slope. These results suggest a gradual decline in the SFR of most galaxies since $z \sim 1-2$.

The specific star formation rate (SSFR), measuring the SFR per unit galaxy stellar mass, allows us to study how the ongoing SF contributes to the mass growth for galaxies of different masses, at different times. Lower-mass galaxies have higher SSFRs than higher-mass galaxies (Feulner et al. 2005; Pérez-González et al. 2005; Zheng et al. 2007; Noeske et al. 2007b), supporting a scenario in which massive galaxies formed most of their stars earlier and on shorter timescales, while less-massive galaxies evolve on longer timescales (“downsizing").

There are several reasons to expect that the SFR-mass and SSFR-mass relations should depend on environment. While fast-acting environmental effects are unlikely to influence the SFR-mass relationship of star-forming galaxies, any physical mechanism slowly affecting the amount of gas available for SF should result in a slowly declining SFR, therefore a different SFR-mass relation with environment. Examples are the loss of halo gas reservoir included in hierarchical galaxy formation models ("strangulation"; Larson et al. 1980; Font et al. 2008; McCarthy et al. 2008), and the interruption of cold gas streams in dense environments that would leave unfueled galaxies to slowly consume their disk gas (Kereš et al. 2005).

In contrast, several works have reported a lack of environmental dependence of the distribution on current SF activity as measured both from optical lines and infrared emission (Balogh et al. 2004b; Rines et al. 2005; Bai et al. 2006, 2007, 2009; but see Wolf et al. 2009), and others have failed to identify large population of galaxies in "transition" from the red sequence to the blue cloud in dense environments (Balogh et al. 2004a; Weinmann et al. 2006; Cassata et al. 2007).

However, the relations between SFR and SSFR with mass have not yet been studied in groups and clusters, which should be the most direct way to clearly discriminate between mass and environmental trends. Should the SFR-mass relation be universal, the evolution of the red galaxy fraction would have a galaxy intrinsic origin, and environmental effects such as strangulation could not be relevant.

In this Letter, we make a first attempt to investigate this issue by studying galaxies in clusters and groups at intermediate redshifts $(0.4<z<0.8)$ using the ESO Distant Cluster Survey (EDisCS) data set and comparing that with field galaxies at similar redshifts.

We adopt $\left(H_{0}, \Omega_{m}, \Omega_{\lambda}\right)=\left(70 \mathrm{~km} \mathrm{~s}^{-1} \mathrm{Mpc}^{-1}, 0.3,0.7\right)$. Values of $M_{*}$ and SFR are based on the Salpeter (1955) initial mass function (IMF) in the range of mass $0.1-125 M_{\odot}$. 


\section{DATA SET}

In our analysis, we use 604 spectroscopically confirmed EDisCS members of 16 clusters with velocity dispersions $\sigma>400 \mathrm{~km} \mathrm{~s}^{-1}$ and 10 groups $\left(150<\sigma<400 \mathrm{~km} \mathrm{~s}^{-1}\right)$ as in Poggianti et al. (2009) (see also Halliday et al. 2004; Milvang-Jensen et al. 2008). In the following, we refer to clusters and groups collectively as "clusters," unless otherwise stated.

Ours is effectively an I-band selected sample with highquality multiband optical and near-IR photometry (White et al. 2005) and spectroscopy (Halliday et al. 2004; Milvang-Jensen et al. 2008), with a $97 \%$ spectroscopic success rate (number of redshifts/number of spectra) at the magnitudes used here.

We estimate galaxy stellar masses using photo- $z$ fitting total absolute magnitudes (Pelló et al. 2009) and the relation between mass-to-light ratio $\left(M / L_{B}\right)$ and rest-frame $(B-V)$ color for solar metallicity from Bell \& de Jong (2001), $\log \frac{M}{L_{B}}=-0.51+$ $1.45(B-V)$. The internal accuracy of the measured masses is $\sim 0.15$ dex. The spectroscopic magnitude limit $(I=23$ at $z=0.8$ and $I=22$ at $z=0.6$ ) corresponds to a mass limit $\log M_{*}=10.8 M_{\odot}$ for galaxies of all colors, and $\log M_{*}=10.5 M_{\odot}$ for blue galaxies (see below for our red/ blue limit)

To estimate SFRs, we use the IR luminosities of Finn et al. (2009). The IR luminosities are derived from Spitzer $24 \mu \mathrm{m}$ imaging, and the observed $24 \mu \mathrm{m}$ flux is converted to total IR luminosity using the models of Dale \& Helou (2002). The IR luminosity is converted to $\mathrm{SFR}_{\mathrm{IR}}$ according to Kennicutt (1998): $\mathrm{SFR}_{\mathrm{IR}}\left(M_{\odot} \mathrm{yr}^{-1}\right)=4.5 \times 10^{-44} \times L_{\mathrm{IR}}\left(\mathrm{erg} \mathrm{s}^{-1}\right)$, assuming that the mid-IR emission of the great majority of distant cluster galaxies is powered by starbursts rather than active galactic nuclei (AGNs), as found by previous studies (Finn et al. 2009 and references therein). The median IR luminosity error is $7 \%$, and is always less than $23 \%$. The $\mathrm{SFR}_{\mathrm{IR}}$ error associated with estimating the IR luminosity from the observed $24 \mu \mathrm{m}$ flux ranges from 5\% to 22\%, depending on the cluster redshift (Finn et al. 2009). The $80 \%$ completeness limit of our Spitzer data corresponds to a $\mathrm{SFR}_{\mathrm{IR}}$ of $\sim 10.3 M_{\odot} \mathrm{yr}^{-1}$ at $z=0.6$ and $\sim 13 M_{\odot} \mathrm{yr}^{-1}$ at $z=0.8$.

We also use the $\mathrm{SFR}_{[\mathrm{O} \text { II }]}$ (Poggianti et al. 2008) from the observed [O II] luminosity using the Kewley et al. (2004) conversion: $\left.\operatorname{SFR}_{[\mathrm{O}} \mathrm{II}\left(M_{\odot} \mathrm{yr}^{-1}\right)=1.26 \times 10^{-41} L_{[\mathrm{O}} \mathrm{II}\right]\left(\mathrm{erg} \mathrm{s}^{-1}\right)$, corrected to our IMF. The SFR ${ }_{[\mathrm{O} \text { II] }}$ detection limit is $\sim 0.3 M_{\odot} \mathrm{yr}^{-1}$. The EDisCS [O II] detections of even weak lines are very robust, having been confirmed by manual inspection of all two-dimensional spectra. The $\mathrm{SFR}_{[\mathrm{OII}}$ errors in $\mathrm{SFR}_{[\mathrm{O} \text { II] }}$ range from $\sim 0.05$ dex to $\sim 0.4$ dex, with a mean error of $\sim 0.1$ dex.

To account for both obscured and unobscured SF, in galaxies with a $24 \mu \mathrm{m}$ detection (all of which have emission lines in their spectra), we use the total $\mathrm{SFR}_{\text {tot }}=\mathrm{SFR}_{\mathrm{IR}}+\mathrm{SFR}_{[\mathrm{OII}}$, without correcting the $\left[\mathrm{O}_{\mathrm{II}}\right]$ estimate for dust extinction. For galaxies without a $24 \mu \mathrm{m}$ detection, we use the $\mathrm{SFR}_{[\mathrm{OII}}$ corrected for dust. The $\left[\mathrm{O}_{\mathrm{II}}\right]$ extinction correction is estimated from the correlation between the uncorrected $\mathrm{SFR}_{[\mathrm{OII}]}$ and $E(B-V)$ observed at low $z: E(B-V)=0.165 \log \left(\mathrm{SFR}_{\left[\mathrm{O}_{\text {II }}\right]}\right)+0.315$ (J. Fritz et al. 2010, in preparation; Kewley et al. 2004). This $E(B-V)$ is derived from the Balmer decrement, thus is appropriate for emission lines. We adopt the Sudžius et al. (1996) Galactic mean interstellar extinction law for which $\frac{A_{3727}}{E(B-V)}=4.749$. Using either the total $24 \mu \mathrm{m}+[\mathrm{O} \mathrm{II}]$ or the dust-corrected [O II] SFRs, the SSFR is simply SSFR $=\frac{\mathrm{SFR}}{M_{*}}$.
Galaxies without a $24 \mu \mathrm{m}$ detection are further divided into red and blue in order to separate those that can be assumed to be powered by SF (blue) from those that could be strongly contaminated by an active galactic nucleus (AGN) (red). Following Noeske et al. (2007a), the color separation is defined by Willmer et al. (2006):

$$
(U-B)_{\text {rest-frame }} \geqslant-0.032\left(M_{B}+21.52\right)+0.454-0.25 .
$$

Based on visual morphological classifications by Desai et al. (2007), blue emission-line (BEL) galaxies and Spitzer-detected galaxies have mostly late-type morphologies ( $\sim 75 \%$ and $\sim 95 \%$, respectively), in agreement with the assumption that they are star forming.

In addition, a fraction of the red emission-line (REL) galaxies could have their [O II] powered by a residual low level of SF, instead of being dominated by an AGN.

In our data set, we currently do not have a way to quantify AGN contamination. External estimates can vary significantly: at low z, Yan et al. (2006) found in their Sloan Digital Sky Survey (SDSS) sample that only $\sim 10 \%$ of red galaxies with $[\mathrm{O}$ II] in emission are characterized by SF. At higher redshifts, Noeske et al. (2007a) found a higher fraction of probable star-formation-dominated REL systems, up to $30 \%$. In broad agreement with this, we find that $32 \%$ of our REL galaxies have late-type morphologies, while only 50\% are ellipticals. This suggests that at least a third of galaxies in this class are indeed star forming. Moreover, analyzing the EDisCS optical spectra, Sánchez-Blázquez et al. (2009) concluded that most of our REL galaxies are dusty and star forming. This agrees with the large population of red star-forming cluster galaxies identified at $z \sim 0.2$ (Wolf et al. 2009).

However, since we cannot be certain of exactly how many red objects should be considered star forming, in our analysis we analyze two different cases: in the first case we assume that the [O II] emission in all red galaxies is dominated by an AGN, and we exclude them from our analysis. In the second case, we include REL galaxies. These cases should bracket the real situation occurring in nature.

\section{COMPARISON WITH THE FIELD}

We compare our results to those for the field at $0.4 \leqslant z \leqslant 0.8$ from Noeske et al. (2007a, 2007b), who studied the SFR and the SSFR in field galaxies from the All-Wavelength Extended Groth Strip International Survey (AEGIS). ${ }^{7}$

Our method to derive SFRs is similar to theirs. For galaxies with robust $24 \mu \mathrm{m}$ detections, they determined the total SFR by summing the SFR derived from $24 \mu \mathrm{m}$ data with that derived from the emission lines uncorrected for extinction, as we do. For galaxies below the $24 \mu \mathrm{m}$ detection limit, they estimated extinction-corrected SFRs from emission-line fluxes using the observed average Balmer decrement, for a fixed $A_{\mathrm{H} \alpha}=1.30$ value. This method overestimates the extinction in galaxies with low SFRs (Noeske et al. 2007a). To avoid systematic effects due to a different dust treatment for the field and cluster galaxies, we de-corrected the AEGIS emission-line data using their value of extinction, then we applied our own method of dust correction to their data points. Our comparison with the

\footnotetext{
7 The Kroupa values (Kroupa 2001) in their paper can be transformed to a Salpeter IMF multiplying by a factor 2 (K. G. Noeske 2009, private communication).
} 

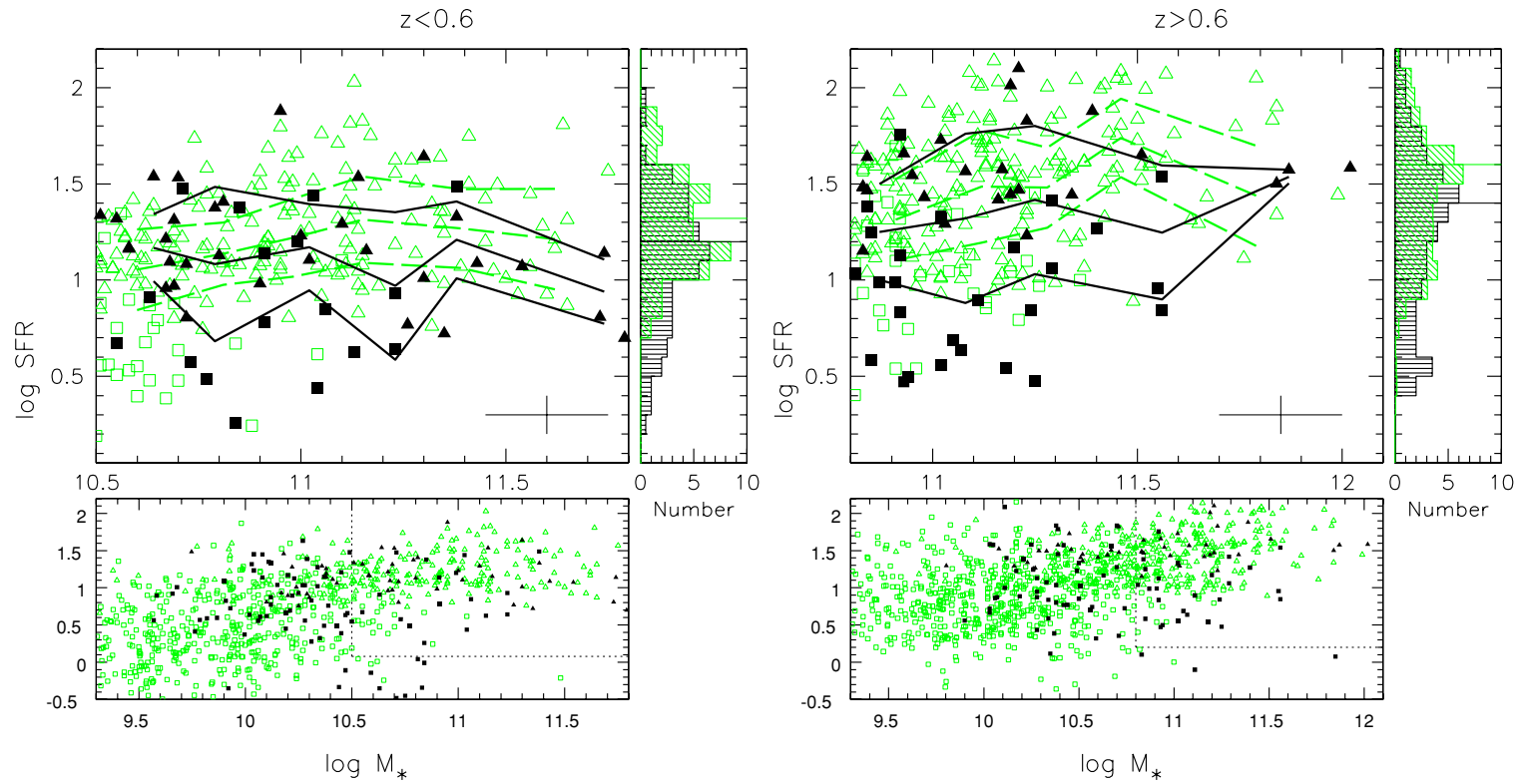

Figure 1. SFR $-M_{*}$ relation of cluster and field galaxies at $z<0.6$ (left panel) and $z>0.6$ (right panel). Only $24 \mu \mathrm{m}+\mathrm{BEL}$ galaxies are plotted. Upper left window: only data points above the completeness limits. Bottom window: all galaxies. Black filled symbols: cluster galaxies (EDisCS). Green empty symbols: field galaxies (AEGIS). Triangles: combined SFRs from MIPS $24 \mu \mathrm{m}$ and emission lines. Squares: [O II] dust-corrected SFRs. In the upper window, lines represent the median and the 25 and 75 percentiles for the clusters (solid black) and field (dashed green). Typical EDisCS error bars are in the bottom right. In the bottom window, lines show the mass and SFR limits. Upper right window: SFR distribution of galaxies above the completeness limits, selecting the same mass distribution in clusters and field, for the average of the 1000 simulations. The number of field galaxies is normalized to the number of cluster galaxies. Black horizontal histogram: EDisCS. Green slanted histogram: AEGIS. Black (EDisCS) and green (AEGIS) solid lines are the mean values of the distributions.
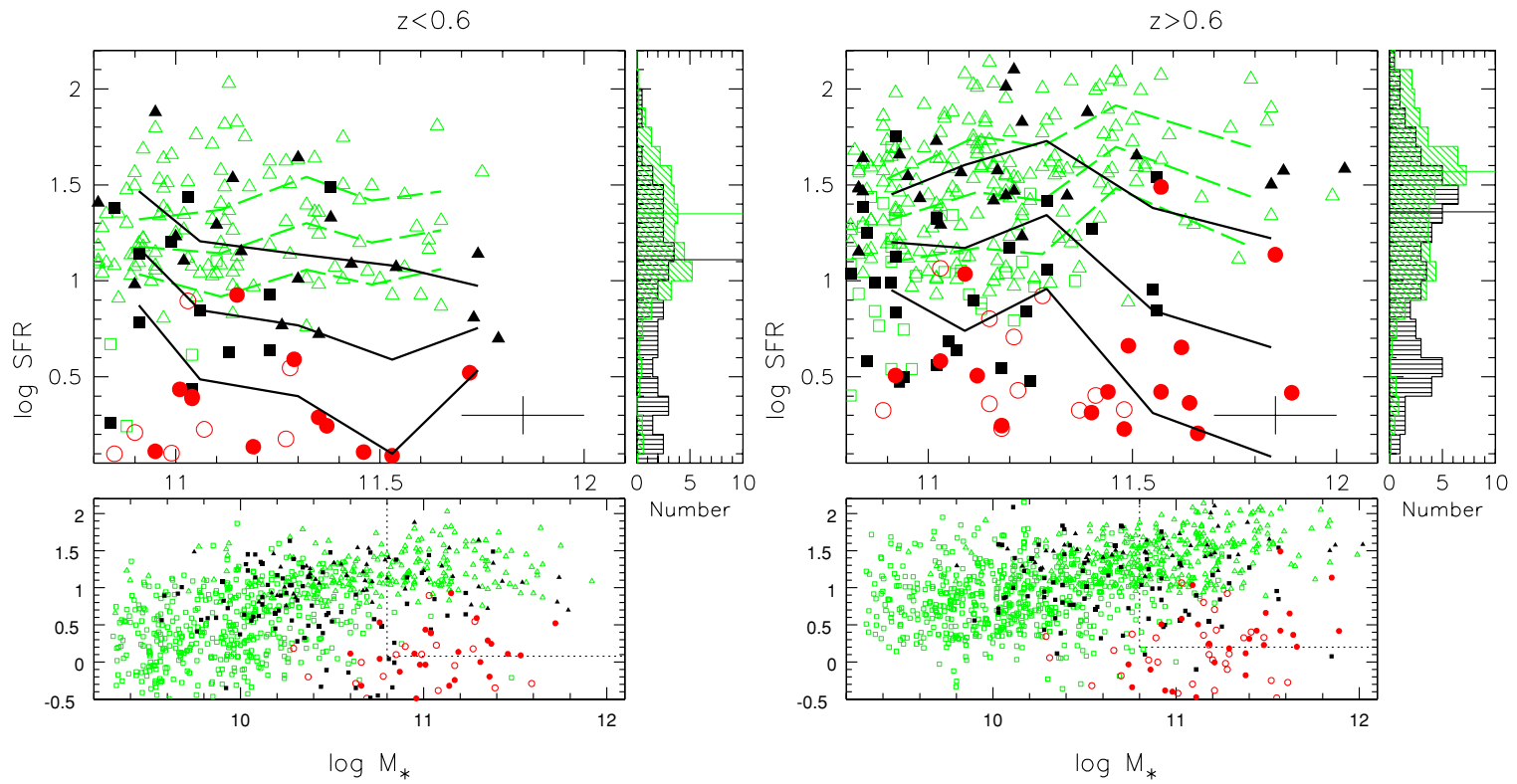

Figure 2. SFR $-M_{*}$ relation of cluster and field galaxies when REL galaxies are included. Red filled circles: cluster REL galaxies. Red empty circles: field REL galaxies. Other symbols as in Figure 1. REL galaxies are included in the histograms.

field is meaningful only for those galaxies with mass and SFR above the highest between our and AEGIS limits, where we are sure that both samples are unbiased. These limits are (1) at $z<0.6, M_{*} \geqslant 10^{10.8} M_{\odot}$ when we consider both blue and red galaxies $\left(10^{10.5} M_{\odot}\right.$ when we do not consider red galaxies), and $\mathrm{SFR}_{\left[\mathrm{OII}_{\text {corr }}\right.} \geqslant 1.2 M_{\odot} \mathrm{yr}^{-1}$; and (2) at $z>0.6, M_{*} \geqslant 10^{10.8} M_{\odot}$ and $\left.\mathrm{SFR}_{[\mathrm{O}}{ }_{\mathrm{II}}\right]_{\text {corr }} \geqslant 1.65 M_{\odot} \mathrm{yr}^{-1}$. Galaxies above our mass limit but below the $\mathrm{SFR}_{[\mathrm{O} \text { II] }}$ limits will be disregarded in our analysis, as they make a negligible contribution to the SFR census. This leaves us with a final cluster sample of 127 galaxies, and a field sample of 426 galaxies.

\section{RESULTS}

We show the SFR-mass relation in different environments in Figure 1, where only $24 \mu \mathrm{m}$ and BEL galaxies are considered star forming. We calculate the median values of SFR and of SSFR, and the 25 and 75 percentiles.

From Figure 1, we note a change with redshift, in both the field and clusters, as the average SFRs at $z \sim 0.7$ are shifted to higher values compared to $z \sim 0.5$ at the same mass.

The main result of Figure 1 is that, at both redshifts, cluster SFRs are on average systematically lower than field SFRs at 

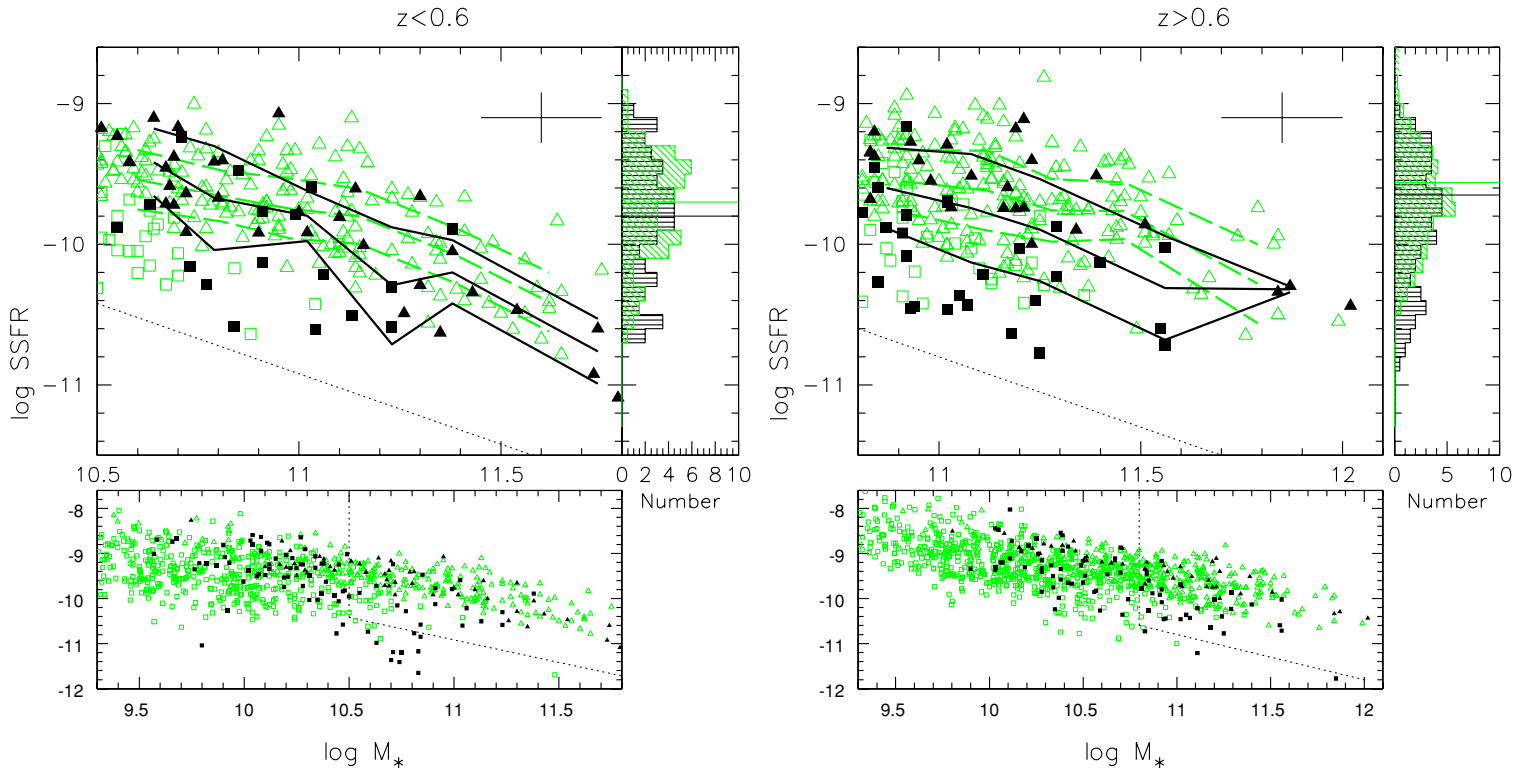

Figure 3. SSFR $-M_{*}$ relation of cluster and field galaxies. Only $24 \mu \mathrm{m}+\mathrm{BEL}$ galaxies are plotted. The dotted lines mark the completeness limits. Symbols are the same as in Figure 1. Typical EDisCS error bars are shown in the top right corner.
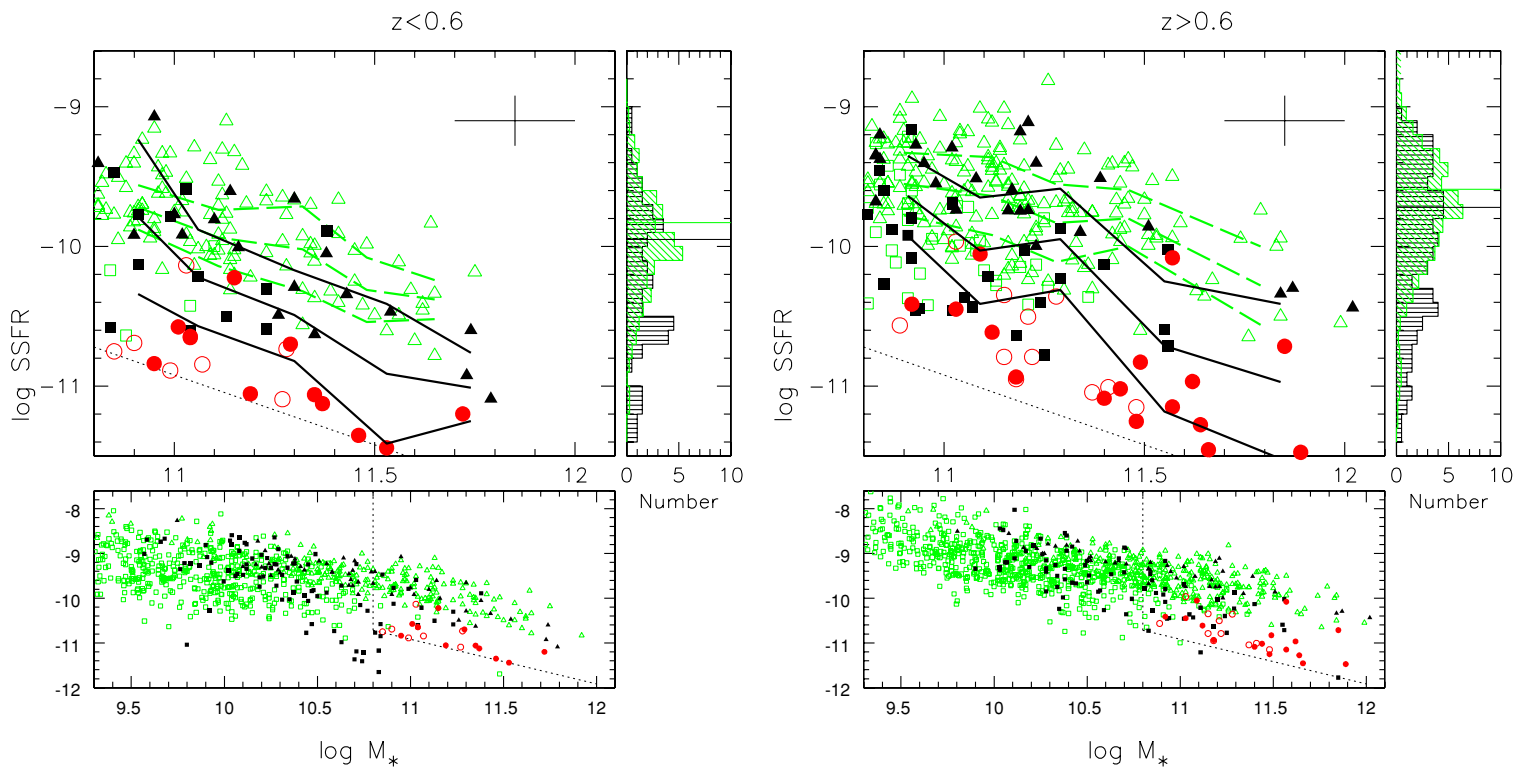

Figure 4. SSFR $-M_{*}$ relation of cluster and field galaxies. Galaxies with $24 \mu \mathrm{m}$ detections and all emission-line galaxies are plotted, regardless of $(U-B)$ color. The dotted lines mark the completeness limits. Symbols are the same as in Figure 2.

the same mass. Clusters have in general lower median SFRs than the field. This is due to a population of cluster galaxies lying below the field 25 percentile, that represent $34 \%$ of the whole cluster population, thus a $\sim 10 \%$ excess of galaxies with "reduced" SFR for their mass.

To avoid the influence of the mass distribution, we performed 1000 Monte Carlo simulations extracting randomly from the field sample a subsample with the same mass distribution as the clusters. The SFR distributions are shown as histograms in Figure 1. At $z<0.6$, due to poor number statistics, ${ }^{8}$ a Kolmogorov-Smirnov (K-S) test cannot reject the null hypothesis of similar SFR distributions in clusters and the field, finding a probability less than $90 \%$ in $54 \%$ of the cases and a probabil-

\footnotetext{
8 Doubling the number of galaxies, differences become significant at $>95 \%$
} in $80 \%$ of the cases. ity $>95 \%$ in $29 \%$ of the simulations. At $z>0.6$, the K-S test rejects the hypothesis of similar cluster and field distributions with a $>95 \%$ probability in $87 \%$ of the simulations.

We note that, although a correlation is evident when considering galaxies over a wide mass range (see bottom windows in Figure 1), the SFR-mass relation is flat above our mass limit. A Spearman test yields a significant positive correlation only for the field at $z>0.6(99.9 \%)$, and no correlation in all other cases.

In Figure 2, we show the results considering also REL galaxies as star forming. We recall that at least for some of them the [O II] emission likely arises from ongoing SF. Now the difference between the field and clusters is more striking, and becomes progressively more pronounced toward more massive galaxies. Fifty percent of the whole cluster population has SFRs below the field 25 percentile, therefore $\sim 25 \%$ have "reduced" 

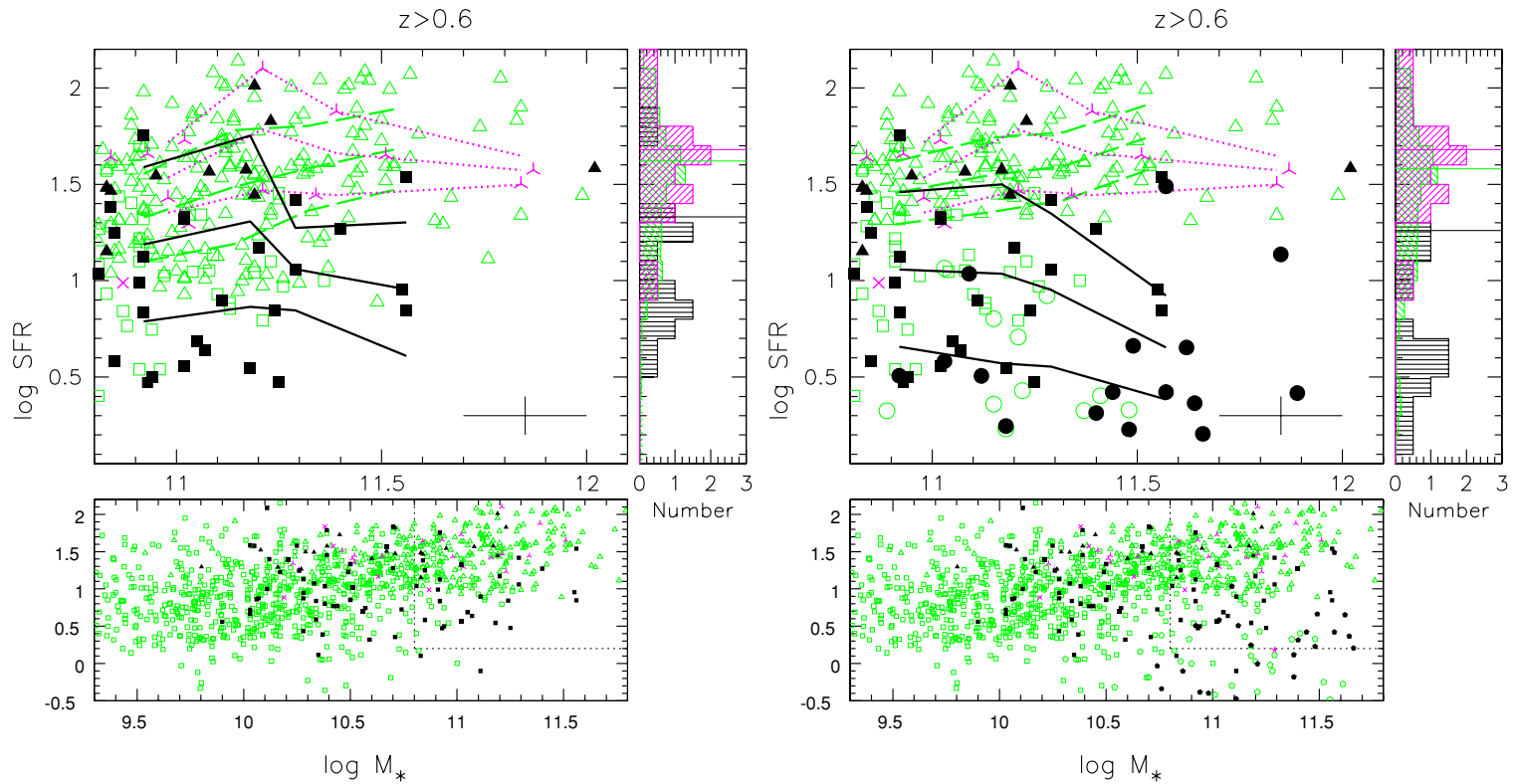

Figure 5. Same as Figure 1, now comparing the SFR- $M_{*}$ relation of cluster, group and field galaxies only at $z>0.6$. Left panel: only $24 \mu \mathrm{m}+\mathrm{BEL}$ galaxies. Right panel: including REL galaxies. Field: green dotted lines and empty symbols as in Figures 1 and 2. Clusters: black solid lines and filled symbols as in Figures 1 and 2. Groups: magenta lines and symbols, skeletal triangles are $24 \mu \mathrm{m}$ detected galaxies, while crosses are BEL galaxies. No REL galaxy is present in groups.

SFRs for their mass compared to the field. The K-S test on massmatched cluster and field samples rejects the null hypothesis of similarity between the two environments with a probability of $100 \%(z<0.6)$ and $>95 \%(z>0.6)$ in all the simulations (see histograms in Figure 2). As before, no SFR-mass correlation is detected by a Spearman test, except for the field at $z>0.6$ at $99.9 \%$.

Our results highlight a change in the SFR-mass relation with environment. To quantify this change, we compute the mean SFR in our mass-matched simulations, therefore removing the effects of different mass distributions. Including both redshift bins, for the sample of $24 \mu \mathrm{m}+\mathrm{BEL}$ galaxies the mean SFR in clusters is $1.35 \pm 0.15$ times lower than in the field. Including REL galaxies, it is $1.63 \pm 0.20$ times lower than the field. ${ }^{9}$

In Figures 3 and 4, we show the results for the SSFR-mass relation. Cluster and field galaxies follow a qualitatively similar decreasing trend of SSFR with mass (Spearman anticorrelation probability always $>99.9 \%$ ), but cluster galaxies tend to have a lower SSFR than field galaxies of similar mass, as expected from the results discussed above. A K-S test confirms this when red galaxies are included (probabilities always $>95 \%$ ). For $24 \mu \mathrm{m}+\mathrm{BEL}$ galaxies, the differences are not statistically significant, with the $\mathrm{K}-\mathrm{S}$ test giving a probability $>95 \%$ only in $53 \%$ of the simulations at $z>0.6$, and only in $4 \%$ of simulations at $z<0.6$. Including both redshift bins, the ratio of average SSFR between the field and clusters above our mass limit is $1.20 \pm 0.14$ for $24 \mu \mathrm{m}+\mathrm{BEL}$ galaxies, and $1.31 \pm 0.17$ for all galaxies.

This result shows that, in all environments, the mass growth rate at a given mass decreases with time (see left and right panels of Figures 3 and 4) and it is lower for higher-mass galaxies (SSFR and mass are anticorrelated). However, a fraction of the star-forming cluster galaxies are building up their stellar mass at a lower rate than field galaxies: $10 \%$ and $30 \%$ of $24 \mu \mathrm{m}+\mathrm{BEL}$ and all galaxies, respectively, lie below the field 25 percentile.

\footnotetext{
9 Errors are computed as bootstrap standard deviations.
}

Moreover, the cluster trends are steeper than the field trends (best-fit slopes differ by $>1 \sigma$ ), again suggesting that SF in more massive galaxies differs more strongly with environment than $\mathrm{SF}$ in lower-mass galaxies.

\subsection{Clusters Versus Groups}

With the aim of investigating whether cluster and group galaxies, separately, differ from the field, we divide the sample into clusters with $\sigma>400 \mathrm{~km} \mathrm{~s}^{-1}$ and groups with $\sigma<$ $400 \mathrm{~km} \mathrm{~s}^{-1}$ (Figure 5). We only consider the highest- $z$ bin, as the lowest- $z$ bin has too few group galaxies to study any trend.

Figure 5 shows that the SF in the cluster environment deviates from the field trend, while group galaxies seem to follow the SFR-mass relation of the field. The $\mathrm{K}-\mathrm{S}$ test performed on mass-matched samples yields a 98\% (without red galaxies) and a $99.9 \%$ (with red galaxies) probability that clusters have a different SFR distribution from both groups and field. In contrast, the test cannot reject the hypothesis that the groups and field have a similar distribution.

Our group data are not sufficient to draw firm conclusions, but, if confirmed, our finding suggests that the group environment is not influential for the link between SF activity and mass, therefore strangulation could not be relevant, at least in groups, and only cluster-specific processes could be important.

Having removed the groups, the mean SFR in clusters at $z>0.6$ is $1.93 \pm 0.02$ (without red) to $2.13 \pm 0.02$ (with red) times lower than in the field.

\section{CONCLUSIONS}

This is the first attempt to establish whether the relation between SF activity and galaxy mass depends on environment. We find that this relation in clusters is significantly different from the field, at all redshifts when REL galaxies are included, and at least at $z>0.6$ for $24 \mu \mathrm{m}+\mathrm{BEL}$ galaxies. Discriminating star forming from AGN red galaxies will allow us to quantify with higher precision the environmental effects. 
The observed differences between the SFRs in clusters and in the field can be considered a lower limit to the real gap. In fact, we are surely considering as cluster members also starforming galaxies that are either in projection or just recently accreted by the cluster and have not been affected yet by its influence.

This result cannot arise from having severely underestimated the dust correction to the $\mathrm{SFR}_{[\mathrm{O} \text { II] }}$. If we were to adjust the [O II] extinction to bring the field and clusters into agreement, many of the [O II] detected sources should be detected at $24 \mu \mathrm{m}$, and they are not.

Environmental differences are detectable thanks to the low completeness limit in SFR reached. With a higher limit, the cluster and field relations would appear compatible. Hence, any comparison of SFR and masses in different environments is meaningful only when all data probe down to low SFR levels.

We conclude that there are significant differences between the SF activity of star-forming galaxies of the same mass in different environments. Clusters, in general, show a lower SF activity than the field, not only because they have a pre-existing large population of early-type galaxies passively evolving since high $z$, but because currently star-forming galaxies host an average lower SFR than their field counterparts of similar mass.

The most straightforward interpretation is that there are environmental effects suppressing SF in clusters. Fast-acting mechanisms would leave the SFR-mass relation unchanged, while processes with a longer timescale would affect it. The most popular long-timescale candidate is strangulation, that, if equally effective in groups and clusters, would be ruled out if the similarity we observe between groups and field will be confirmed by larger studies. Even ram pressure stripping, which acts on a short timescale (Bekki 2009), may leave residual gas and low SFRs.

As an alternative to environmental mechanisms, it is possible that other galaxy intrinsic properties besides mass (e.g., the morphological distribution) influence the SF history and vary systematically with environment.

In clusters, we are observing a population of galaxies in transition from being blue star forming to red passively evolving, while such a population is much less noticeable in the field and perhaps also in groups.

We thank the anonymous referee for his/her useful remarks. We thank Kai Noeske and the AEGIS collaboration for provid- ing us their data and support. We acknowledge financial support from ASI contract I/016/07/0.

\section{REFERENCES}

Bai, L., Rieke, G. H., Rieke, M. J., Christlein, D., \& Zabludoff, A. I. 2009, ApJ, 693, 1840

Bai, L., Rieke, G. H., Rieke, M. J., Hinz, J. L., Kelly, D. M., \& Blaylock, M. 2006, ApJ, 639, 827

Bai, L., et al. 2007, ApJ, 664, 181

Balogh, M. L., Baldry, I. K., Nichol, R., Miller, C., Bower, R., \& Glazebrook, K. 2004a, ApJ, 615, L101

Balogh, M., et al. 2004b, MNRAS, 348, 1355

Bekki, K. 2009, MNRAS, 399, 2221

Bell, E. F., \& de Jong, R. S. 2001, ApJ, 550, 212

Brinchmann, J., Charlot, S., White, S. D. M., Tremonti, C., Kauffmann, G. Heckman, T., \& Brinkmann, J. 2004, MNRAS, 351, 1151

Cassata, P., et al. 2007, ApJS, 172, 270

Daddi, E., et al. 2007, ApJ, 670, 156

Dale, D. A., \& Helou, G. 2002, ApJ, 576, 159

Desai, V., et al. 2007, ApJ, 660, 1151

Elbaz, D., et al. 2007, A\&A, 468, 33

Feulner, G., Gabasch, A., Salvato, M., Drory, N., Hopp, U., \& Bender, R. 2005, ApJ, 633, L9

Finn, R., et al. 2009, ApJ, submitted

Font, A. S., et al. 2008, MNRAS, 389, 1619

Halliday, C., et al. 2004, A\&A, 427, 397

Kennicutt, R. C., Jr. 1998, ARA\&A, 36, 189

Kereš, D., Katz, N., Weinberg, D. H., \& Davé, R. 2005, MNRAS, 363, 2

Kewley, L. J., Geller, M. J., \& Jansen, R. A. 2004, AJ, 127, 2002

Kroupa, P. 2001, MNRAS, 322, 231

Larson, R. B., Tinsley, B. M., \& Caldwell, C. N. 1980, ApJ, 237, 692

McCarthy, I. G., Frenk, C. S., Font, A. S., Lacey, C. G., Bower, R. G., Mitchell, N. L., Balogh, M. L., \& Theuns, T. 2008, MNRAS, 383, 593

Milvang-Jensen, B., et al. 2008, A\&A, 482, 419

Noeske, K. G., et al. 2007a, ApJ, 660, L43

Noeske, K. G., et al. 2007b, ApJ, 660, L47

Pannella, M., et al. 2009, ApJ, 698, L116

Pelló, R., et al. 2009, arXiv:0910.5418

Pérez-González, P. G., et al. 2005, ApJ, 630, 82

Poggianti, B. M., et al. 2008, ApJ, 684, 888

Poggianti, B. M., et al. 2009, ApJ, 693, 112

Rines, K., Geller, M. J., Kurtz, M. J., \& Diaferio, A. 2005, AJ, 130, 1482

Salpeter, E. E. 1955, ApJ, 121, 161

Sánchez-Blázquez, P., et al. 2009, A\&A, 499, 47

Sudžius, J., Bobinas, V., \& Raudeliu, S. 1996, Mem. Soc. Astron. Ital., 67, 1079

Weinmann, S. M., van den Bosch, F. C., Yang, X., \& Mo, H. J. 2006, MNRAS 366,2

White, S. D. M., et al. 2005, A\&A, 444, 365

Willmer, C. N. A., et al. 2006, ApJ, 647, 853

Wolf, C., et al. 2009, MNRAS, 393, 1302

Yan, R., Newman, J. A., Faber, S. M., Konidaris, N., Koo, D., \& Davis, M. 2006, ApJ, 648, 281

Zheng, X. Z., Bell, E. F., Papovich, C., Wolf, C., Meisenheimer, K., Rix, H.-W., Rieke, G. H., \& Somerville, R. 2007, ApJ, 661, L41 